\title{
Conductivity across random barrier distribution as origin of large low-frequency dielectric peak in perovskite crystals and ceramics
}

\section{Authors: V.Hugo Schmidt, G.F. Tuthill, C.-S. Tu, T.V. Schogoleva, and S.C. Meschia}

NOTICE: this is the author's version of a work that was accepted for publication in Journal of Physics and Chemistry of Solids. Changes resulting from the publishing process, such as peer review, editing, corrections, structural formatting, and other quality control mechanisms may not be reflected in this document. Changes may have been made to this work since it was submitted for publication. A definitive version was subsequently published in Journal of Physics and Chemistry of Solids, [VOL\# 57, ISSUE\# 10, (October 1996)] DOI\# 10.1016/0022-3697(96)80020-5

V.H. Schmidt, G.F. Tuthill, C.-S. Tu, T.V. Schogoleva, and S.C. Meschia, "Conductivity across random barrier distribution as origin of large low-frequency dielectric peak in perovskite crystals and ceramics," Journal of Physics and Chemistry of Solids 57, 1493-1497 (1996). 


\title{
CONDUCTIVITY ACROSS RANDOM BARRIER DISTRIBUTION AS ORIGIN OF LARGE LOW-FREQUENCY DIELECTRIC PEAK IN PEROVSKITE CRYSTALS AND CERAMICS
}

\author{
V. HUGO SCHMIDT, GEORGE F. TUTHILL, CHI-SHUN TU, \\ TATYANA V. SCHOGOLEVA and STEVEN C. MESCHIA \\ Department of Physics, Montana State University, Bozeman, MT 59717, U.S.A.
}

(Received 6 June 1995; accepted 14 August 1995)

\begin{abstract}
Several perovskite crystals and ceramics show very large dielectric $\left(\epsilon^{\prime}\right)$ peaks at high temperature $T$ and low frequency $f$. In some cases these peaks are in the cubic phase far above any ferroelectric transition. Even at the peaks, the lossy part $\epsilon^{\prime \prime}$ is larger than the real part $\epsilon^{\prime}$. The $\epsilon^{\prime}$ vs $T$ curves for different $f$ follow the same d.c. (low- $f$ ) envelope down to some $T(f)$ below which the curve for that $f$ falls below the envelope. Similarly, the conductivity (or $\epsilon^{\prime \prime}$ ) data show d.c. and a.c. (high-frequency) envelopes for which data at different $f$ overlap. As a first approximation to a crystal with random barriers impeding conductivity, a model with barriers $B$ (in $T$ units) every lattice constant $a=4 \AA$ and barriers $B+\Delta$ every distance $d$ is assumed. The model is fit to permittivity and conductivity data for a strontium titanate single crystal, and a good qualitative fit is obtained.
\end{abstract}

Keywords: A. ceramics, A. oxides, D. dielectric properties, D. electrical conductivity.

\section{INTRODUCTION}

The barium titanate family of perovskite ferroelectric, relaxor ferroelectric, and related crystals and ceramics is one of the oldest, and is the most technically important family of ferroelectric materials. Its ferroelectric transitions were thought to be primarily of the displacive type, with some quasistatic disorder introduced by the random unlike charge cations in the relaxor ferroelectric crystals. Recently it was found [1] that even in $\mathrm{PbTiO}_{3}$, which was considered to have a purely displacive transition, there are polar clusters persisting well above the ferroelectric transition temperature $T_{\mathrm{c}}$.

Over some years there has been data collected on conductivity and permittivity in perovskite crystals and ceramics. Much of this is concerned with ferroelectric and other transitions in pure and mixed (relaxor ferroelectric) crystals. In addition to anomalies connected with these transitions, many workers have seen dispersive dielectric anomalies at high temperature and low frequency. In some cases, conductivity data were obtained as well [2-5]. These results were interpreted in a variety of ways, in some cases as being associated with reorienting dipoles [3,5], and in other cases as effects of blocking surface layers $[2,4]$.

\section{PHENOMENA OBSERVED}

There are several characteristic features of the above results.
(1) The typical conductivity $\left(\sigma^{\prime}\right)$ vs temperature plot shows a high-frequency envelope on which data from a wide frequency range overlap, as seen in Fig. 1 for $\mathrm{SrTiO}_{3}$ [2]. This envelope shows nearly Arrheniustype thermally activated behavior.

(2) The conductivity vs temperature plot also shows a low-frequency envelope for which data for various frequencies overlap. This envelope also nearly obeys the Arrhenius law, but with a higher activation energy than for the high-frequency envelope.

(3) For available data, the low-frequency and highfrequency envelopes do not meet, but if extrapolated towards higher temperatures on an Arrhenius plot of $\log$ conductivity vs $1 / T$ they meet at a finite temperature.

(4) The conductivity data show frequency dispersion, in that the curves for various frequencies cross over from the high-frequency to the low-frequency envelopes at different locations for different frequencies.

(5) The dielectric permittivity $\left(\epsilon^{\prime}\right)$ vs temperature plots show dispersion, in that the permittivity peak moves to a lower temperature and becomes higher as frequency decreases, as seen in Fig. 2 for $\mathrm{SrTiO}_{3}$ [2]. Available data do not go to a low enough frequency to show saturation of this peak height increase.

(6) The dielectric permittivity curves for different frequencies follow a common envelope at a high temperature, with the highest frequencies peeling off the curve first as $T$ decreases, so this can be called the low-frequency envelope. 


\section{$\mathrm{SrTiO}_{3}$}

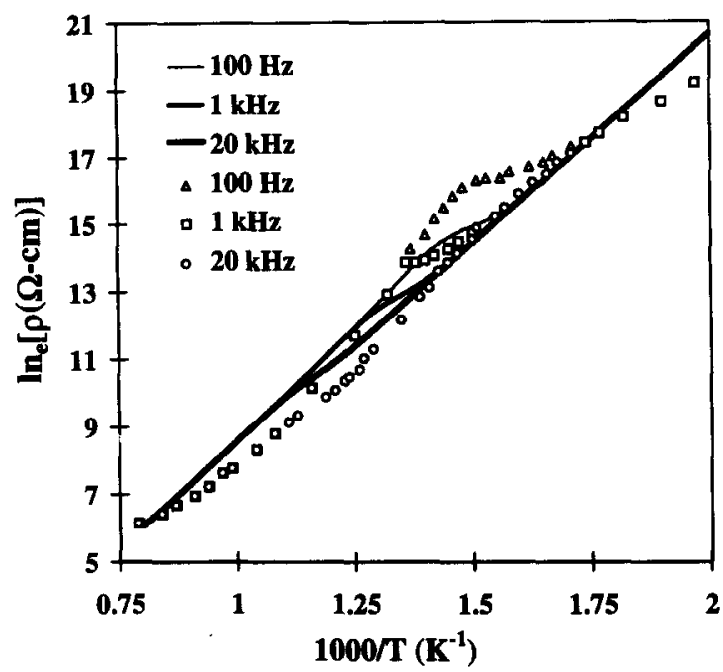

Fig. 1. Fit of our model (lines) to $\mathrm{SrTiO}_{3}$ single crystal resistivity data (symbols) (plotted as $\mathbf{l n}_{e}$ ) from Ref. [2] Model parameters are $B=13000 \mathrm{~K}, \Delta=4150 \mathrm{~K}$, and $d=50 \mathrm{~nm}$.

(7) If the real part $\epsilon^{\prime}$ of the permittivity is converted to the imaginary part $\sigma^{\prime \prime}$ of the conductivity by the relation $\sigma^{\prime \prime}=\epsilon_{0} \epsilon^{\prime} \omega$, there is a high-frequency envelope for $\sigma^{\prime \prime}$ from which the low-frequency curves peel off first as temperature increases.

(8) If a plot of logarithm of peak permittivity $\epsilon^{\prime}$ vs $1 / T$ at the peak is made for various frequencies, it obeys an Arrhenius relation, as seen by us for the relaxor ferroelectric NBT $\left(\mathrm{Na}_{1 / 2} \mathrm{Bi}_{1 / 2} \mathrm{TiO}_{3}\right)$ [5]. If $\epsilon^{\prime \prime}$ values at the same $f$ and $T$ values are also plotted, they obey an Arrhenius relation with similar activation energy. The $\epsilon^{\prime \prime}$ values are consistently higher than the $\epsilon^{\prime}$ values for various crystals and ceramics.

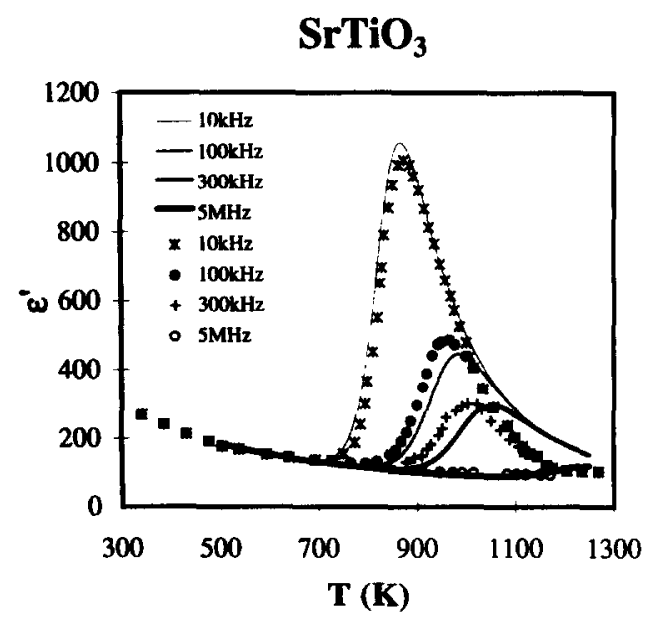

Fig. 2. Fit of our model (lines) to $\mathrm{SrTiO}_{3}$ single crystal permittivity data (symbols) from Ref. [2]. Model parameters are $B=13000 \mathrm{~K}, \Delta=4150 \mathrm{~K}$, and $d=50 \mathrm{~nm}$.
(9) If the Arrhenius-law envelope is subtracted from the $\epsilon^{\prime \prime}$ data, the remaining $\epsilon^{\prime \prime}$ values plotted against $\epsilon^{\prime}$ in a Cole-Cole diagram show nearly monodispersive Debye behavior, namely a semicircle with center only slightly below the $\epsilon^{\prime}$ axis. Such behavior was seen by us in NBT [5].

In our investigation of permittivity and conductivity of the relaxor ferroelectric NBT [5], the dispersive behavior of $\epsilon^{\prime}$ in the temperature range of the orthorhombic phase led us to believe that this large lowfrequency dielectric peak was another feature of NBT's relaxor ferroelectric behavior. We attributed the similarity of conductivity and peak dielectric activation energies to the dipolar cluster reorientation rate being determined by rearrangement of the carriers which provide the conductivity mechanism. We later learned that similar phenomena occur in a wide variety of perovskite crystals and ceramics, in some cases far above any ferroelectric transition. We compared plots of these phenomena with those seen by us in NBT, and attributed these phenomena to phaseshifted electrical conductivity [6].

In ordinary conductivity there is no associated permittivity or imaginary part of the conductivity. This is true even if the mobility is thermally activated in the sense that the carriers must hop over intrinsic barriers (all of equal height) in going from one lattice site to the next. In order to produce an associated permittivity, there must be extrinsic barriers, higher than the intrinsic barriers, spaced farther apart than a lattice spacing. These barriers could be at the electrodes, $[2,4]$ or at internal grain boundaries (giving the Maxwell-Wagner effect).

\section{MODEL}

As a first attempt at a model which can predict the above behavior, we choose one which has barriers $B$ every lattice spacing $a$, and higher barriers $B+\Delta$ every distance $d$, as shown in Fig. 3. This distance $d$ could represent the width of the crystal, or if internal barriers are dominant, the typical distance between

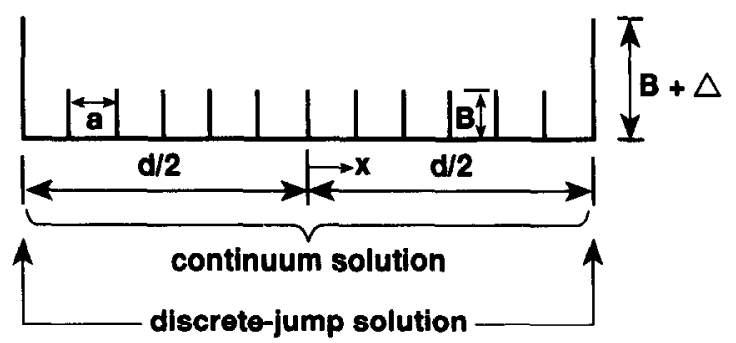

Fig. 3. Illustration of 2-barrier-height model showing intrinsic barriers $B$ at spacing $a$, and extrinsic barriers $B+\Delta$ at spacing $d$. 
such barriers. If the barriers are internal, all phenomena such as current density and electric field are periodic in space with period $d$. A mathematically similar model, but with $\Delta \rightarrow \infty$ and $d$ being the crystal width, was developed by Coelho [7] and was applied by Bidault et al. [4] to $\mathrm{BaTiO}_{3}$. That model, which assumes completely blocking electrodes at the crystal surfaces, gives very different results than our partially-blocking barriers model.

The high-frequency behavior, which is characterized by the high-frequency conductivity envelope and by the Curie-Weiss law permittivity without the dispersive high-temperature permittivity peaks, is determined by the intrinsic barrier height $B$ and the carrier concentration $n$. Accordingly all the remaining eight features listed above must be predicted for various frequencies by only two parameters, $\Delta$ and $d$.

To preview our analysis method, we treat the spaces of width $d$ between the higher barriers in the continuum approximation, but treat the hopping across the higher barriers as a discrete process. The overall solution is achieved by requiring that the current densities from the discrete and continuous solutions match, and that the net charge density vanishes at the mid-point between the higher barriers.

The continuum solution is achieved by means of three equations. First there is the equation for current density $J(x, t)$, with the 'high-frequency conductivity' $\sigma_{\infty}$ term which gives the effect of the intrinsic barriers, and the 'diffusion' term which accounts for the backdiffusion tendency of the carriers which pile up against the higher barriers. Second, there is Gauss' Law which modifies the applied electric field to add in the field from the excess or missing carrier density on opposite sides of the higher barriers. Third, there is the continuity equation which relates spatial dependence of current density to time variation of charge density $\rho$.

The equations solved in this manner provide the current density $J(x, t)$ and the electric field $E(x, t)$ for a sinusoidal applied voltage. These are related to the measured current $I$ and voltage $V$ by $I=J_{\mathrm{t}} S=$ $(J+\partial D / \partial t) S$ and $V=\langle E\rangle l$, where $S$ and $l$ are crystal area and thickness, $\langle E\rangle$ is defined in eqn (15), and $D=\epsilon_{\infty} E$ with $\epsilon_{\infty}$ defined below eqn (5). The conductivity $\sigma$ is given by $\sigma=J_{t} /\langle E\rangle$.

We now give the details of the model calculation. First we find an expression for the high-frequency conductivity envelope $\sigma_{\infty}$. We assume extrinsic conductivity (the usual type for perovskites) with a temperature-independent carrier density $n$ and carrier charge $q$. The observed $\sigma_{\infty}$ activation energy then comes from the intrinsic barriers of height $B$ (in temperature units) which occur every distance $a$, where $a$ is of order of a lattice constant. We obtain the usual expression for hopping conductivity
$\sigma_{\infty}=J / E$, taking into account that the number of carriers per unit area in a unit-cell layer on either side of a barrier layer is na and their attempt frequency for crossing the barrier is $\nu$ :

$$
\begin{aligned}
\sigma_{\infty}= & E^{-1} q \nu n a\left\{\operatorname { e x p } \left[\left(-k B+\frac{1}{2} q E a\right) / k T\right.\right. \\
& \left.-\exp \left[\left(-k B-\frac{1}{2} q E a\right) / k T\right]\right\} \\
& \simeq\left(q^{2} \nu n a^{2} / k T\right) \mathrm{e}^{-B / T}
\end{aligned}
$$

To reduce the number of adjustable parameters, we assume $\nu=k T / h$ ( $h$ is Planck's constant) which is not quite correct above the Debye temperature (where we are for this high-temperature behavior). However, this assumption eliminates the pre-exponential $T$-dependence, which in any case is hard to detect in the presence of a large activation temperature $B$. We also assume $q=2 e$ (twice the proton charge) which is the value consistent with oxygen vacancy carriers and we take $a$ as $0.4 \mathrm{~nm}$, the typical perovskite lattice constant. Any inaccuracy in these values of $\nu, q$ or $a$ will be reflected in inaccuracy in the $n$ value found from fitting our final expression

$$
\sigma_{\infty}=\left(n q^{2} a^{2} / h\right)-e^{B / T}
$$

to the high-frequency conductivity data for a given sample.

We now turn to the model predictions for lowerfrequency behavior for which the higher barriers $B+\Delta$ must be taken into account. To provide the continuum differential equation for the net charge density $\rho$, we first need the equation for current density $J$ :

$$
J=\sigma_{\infty} E-\sigma_{\infty} \tau \partial \rho / \partial x
$$

where $\tau \equiv k T / q^{2} n, k$ is Boltzmann's constant, $T$ is temperature, $q$ is carrier charge, and $\sigma_{\infty}$ is the highfrequency conductivity found above. The first and second terms are the conduction and diffusion terms discussed above.

To eliminate $E$ from this equation, we divide by $\sigma_{\infty}$, take $\partial / \partial x$ and put $\partial E / \partial x$ by itself, as

$$
\partial E / \partial x=(\partial J / \partial x) / \sigma_{\infty}+\tau \partial^{2} \rho / \partial x^{2}
$$

Then we use Gauss' law, which for our planar geometry yields

$$
\partial E / \partial x=\rho / \epsilon_{\infty^{\prime}}
$$

where $\epsilon_{\infty} \equiv \epsilon_{0} \epsilon_{\mathrm{b}}$. Here $\epsilon_{0}$ is the MKS permittivity constant $8.85 \times 10^{-12} C^{2} / \mathrm{Nm}^{2}$ and $\epsilon_{\mathrm{b}}$ is the background permittivity given by the Curie-Weiss law

$$
\epsilon_{\mathrm{b}}=C /\left(T-T_{0}\right),
$$

in which $C$ and $T_{0}$ are the Curie-Weiss constant and Curie-Weiss temperature, respectively, obtained by fitting the high-frequency permittivity. 
By equating the right sides of eqns (4) and (5), we obtain an equation from which we can eliminate $J$ by using the continuity equation, which for planar geometry is

$$
\partial J / \partial x=-\partial \rho / \partial t
$$

Upon making this substitution for $\partial J / \partial x$, dividing by $\tau$, and rearranging, we obtain a differential equation for $\rho$ which is valid in the range $-d / 2<x<d / 2$ between the higher barriers:

$$
\partial^{2} \rho / \partial x^{2}-\rho / \epsilon_{\infty} \tau-\left(1 / \sigma_{\infty} \tau\right) \partial \rho / \partial t=0 .
$$

Fortunately, the $x$ and $t$ variables are separable in the solution

$$
\rho(x, t)=f(x) \mathrm{e}^{\mathrm{i} \omega t},
$$

for which the $f(x)$ equation becomes

$$
\partial^{2} f / \partial x^{2}-\alpha^{2} f=0 ; \quad \alpha^{2} \equiv 1 / \epsilon_{\infty} \tau+\mathrm{i} \omega / \sigma_{\infty} \tau
$$

The solution for $f(x)$ is

$$
\begin{aligned}
f(x)= & A \mathrm{e}^{\alpha x}+B \mathrm{e}^{-\alpha x} \\
\alpha= & {\left[\left(1 / \epsilon_{\infty} \tau\right)^{2}+\left(\omega / \sigma_{\infty} \tau\right)^{2}\right]^{\frac{1}{4}} } \\
& \exp \left[\frac{1}{2} \operatorname{itan}^{-1}\left(\omega \epsilon_{\infty} / \sigma_{\infty}\right] .\right.
\end{aligned}
$$

For an overall neutral crystal we expect $\rho(x=0)=0$ at the mid-point between higher barriers, so $B=-A$ in eqn (9).

Given $A$, we can find the current $J( \pm d / 2)$ across the high barriers. Then with $J( \pm d / 2)$ known, we can use eqn (3) to find $E( \pm d / 2)$. We then find $E(x)$ from eqn (5), and integrate $E(x)$ and divide by $d$ to find the average $\langle E\rangle$. The conductivity then is given by $\sigma(\omega)=\sigma^{\prime}+i \sigma^{\prime \prime}=J_{t}$. We now go through these steps.

The difference in carrier density across the higher barriers is $\rho(d / 2)-\rho(-d / 2)=2 \rho(d / 2)$. The difference in density per unit area (in the unit cells next to the higher barriers) is $2 a \rho(d / 2)$. These carriers have an attempt frequency $\nu$ and a success probability $\exp [-(B+\Delta) / k T]$ of crossing the barriers. Using eqn (11), the current density at the barrier is (omitting the $\mathrm{e}^{\mathrm{i} \omega t}$ factor)

$$
\begin{aligned}
J(d / 2)= & \left\{2 \nu a A\left(\mathrm{e}^{\alpha d / 2}-\mathrm{e}^{-\alpha d / 2}\right) \exp \right. \\
& {\left.[-B / T]+\sigma_{\infty} E(d / 2)\right\} \mathrm{e}^{-\Delta / T} }
\end{aligned}
$$

Then using eqn (3) and differentiating eqn (11), the field at the high barriers is (omitting $\mathrm{e}^{\mathrm{i} \omega t}$ )

$$
\begin{aligned}
E(d / 2)= & \left(2 \nu a A / \sigma_{\infty}\right)\left(\mathrm{e}^{\alpha d / 2}-\mathrm{e}^{-\alpha d / 2}\right) \exp \\
& {[-(B+\Delta) / T]+E(d / 2) \mathrm{e}^{-\Delta / T} } \\
& +\pi \alpha A\left(\mathrm{c}^{\alpha d / 2}+\mathrm{e}^{-\alpha d / 2}\right)
\end{aligned}
$$

But $\sigma_{\infty}=\left(\nu a^{2} / \tau\right) e^{-B / T}$, so we obtain

$$
\begin{aligned}
E(+d / 2)= & \left\{2 a^{-1}\left(\mathrm{e}^{\alpha d / 2}-\mathrm{e}^{-\alpha d / 2}\right) \mathrm{e}^{-\Delta / T}+\alpha\right. \\
& \left.\left(\mathrm{e}^{\alpha d / 2}+\mathrm{e}^{-\alpha d / 2}\right)\right\} \tau A /\left(1-\mathrm{e}^{-\Delta / T}\right),
\end{aligned}
$$

where we see that $A$ must vanish if $\Delta=0$, as expected because the higher barriers causing the net charge density become the same height as the other barriers.

To find the average $\langle E\rangle$, we first integrate eqn (5) and add $E(-d / 2)$ from eqn (14) to find $E(x)$. Then we integrate $E(x)$ and divide by $d$ to find $\langle E\rangle$ :

$$
\begin{aligned}
E(x)= & E(-d / 2)+\int_{-d / 2}^{x} \epsilon_{\infty}^{-1} A\left(\mathrm{e}^{\alpha x}-e^{-\alpha x}\right) \mathrm{d} x \\
= & E(-d / 2)+\left(\epsilon_{\infty} \alpha\right)^{-1} A\left(\mathrm{e}^{\alpha x}+\mathrm{e}^{-\alpha x}\right. \\
& \left.-\mathrm{e}^{\alpha d / 2}-\mathrm{e}^{-\alpha d / 2}\right), \\
\langle E\rangle= & d^{-1} \int_{-d / 2}^{d / 2} E(x) \mathrm{d} x
\end{aligned}
$$

By combining eqns (12) and (15) and performing considerable algebraic and trigonometric manipulation we obtain

$$
\begin{aligned}
\sigma(\omega)= & \sigma^{\prime}(\omega)+i \sigma^{\prime \prime}(\omega) \\
= & \frac{\sigma_{\infty}\left[b \cosh b+r \sinh b+i \tan w\left(b e^{\Delta / T} \cosh b+r \sinh b\right)\right]}{b \mathrm{e}^{\Delta / T} \cosh b+r \sinh b-\left(\cos ^{2} w-i \sin w \cos w\right)} \\
& \times \frac{1}{\left(\mathrm{e}^{\Delta / T}-1\right)(b \cosh b-\sinh b)}
\end{aligned}
$$

where $b=\alpha d / 2, r=d / a$, and $w=\tan ^{-1}\left(\omega \epsilon_{\infty} / \sigma_{\infty}\right)$.

\section{FIT TO EXPERIMENTAL RESULTS}

Computer analysis of the above expressions can encounter underflow problems for some ranges of variables, but we were able to circumvent these. We tried a wide variety of sets of $B, \Delta$, and $d$ to fit both the permittivity and resistivity data [2] for $\mathrm{SrTiO}_{3}$. The 'best fit' depends on the weight given to fitting different features of the curves. We are most satisfied with the fit shown in Figs 1 and 2. The main discrepancy in Fig. 1 is that the dispersion is not as strong as observed experimentally. In Fig. 2 some of the calculated permittivity peaks occur at higher temperatures than observed. We emphasize that only one set of three parameters is used to fit both curves. In reality, the barrier $B=13,000 \mathrm{~K}$ which occurs at every site is fixed by the a.c. conductivity envelope, leaving only the additional barrier height $\Delta=4150 \mathrm{~K}$ and its spacing $d=50 \mathrm{~nm}$ as fitting parameters to fit all the features of both the conductivity and permittivity. We consider this a good qualitative fit for a model with only two free parameters.

The spacing $d=50 \mathrm{~nm}$ of the higher barriers is much less than the crystal thickness of $1.02 \mathrm{~mm}$, so these barriers do not appear to be associated with partially blocking contacts. 
This fit could be improved by including a barrier distribution with more than two heights. Such fitting could be called 'conductivity spectroscopy' and constitutes a new analysis technique, that of obtaining barrier distributions from conductivity data.

\section{CONCLUSIONS}

From this success in fitting the $\mathrm{SrTiO}_{3}$ data, we expect it will be possible to explain the main features of both the dielectric permittivity and the electrical conductivity at high temperature in a variety of perovskite crystals and ceramics, including relaxor ferroelectrics, by a model in which the carriers encounter barriers with a height distribution.

Strontium titanate [2] provided the simplest fitting problem, because the background permittivity obeys the simple Curie $C / T$ law in the high-temperature range of interest. Next in difficulty will be barium titanate [4], with its highest-temperature transition well below the range of interest, so that a CurieWeiss $C /\left(T-T_{0}\right)$ background permittivity can be added to that obtained from the barrier model. A greater challenge will be $\mathrm{PbTiO}_{3}$ crystals and related crystals and ceramics [3] which have high-temperature transitions. Even more difficult to analyze are relaxor ferroelectrics such as NBT $[5,6]$. Its highest transition, besides being at high temperature, is smeared out for poorly-understood reasons so that it is difficult to separate the barrier-induced permittivity from the background contribution. Fitting of the model to crystals and ceramics such as these is in progress.
On the experimental side, we emphasize the importance of making conductivity as well as permittivity measurements on crystals and ceramics such as these. Specifically, one should plot the lossy part $\epsilon^{\prime \prime}$ as well as the real part $\epsilon^{\prime}$ of the permittivity on the same scale, so that the situation $\epsilon^{\prime \prime}>\epsilon^{\prime}$ symptomatic of phaseshifted conductivity is easy to detect.

The measurement range should be extended to lower frequencies and lower temperatures, to look for saturation of the permittivity peak. In all materials studied so far, this peak continues to increase in height and it occurs at a lower temperature, as frequency is lowered. However, frequencies below $20 \mathrm{~Hz}$ have not yet been employed. The 2-barrier-height model predicts a yet unseen saturation.

Acknowledgements-Professors L. E. Cross and D. M Smyth are thanked for helpful discussions. This work was supported by NASA Grant NCCW-0058.

\section{REFERENCES}

1. Sicron N., Ravel B., Yacoby Y., Stern E. A., Dogan F. and Rehr J. J., Phys. Rev. B50, 13168 (1994).

2. Stumpe R., Wagner D. and Bäuerle D., Phys. stat. sol. (a) 75, 143 (1983).

3. Kuwabara M., Goda K. and Oshima K., Phys. Rev. B42, 10012 (1990).

4. Bidault O., Goux P., Kchikech M., Belkaoumi M. and Maglione M., Phys. Rev. B49, 7868 (1994).

5. Tu C.-S., Siny I. G. and Schmidt V. H., Phys. Rev. B49, 11550 (1994).

6. Schmidt V. H., Tu C.-S. and Siny I. G., IEEE Proc. of Int. Symp. Applications of Ferroelectrics (ISAF94), p. 45 (1995).

7. Coelho R., Physics of Dielectrics. Elsevier, New York (1979). 